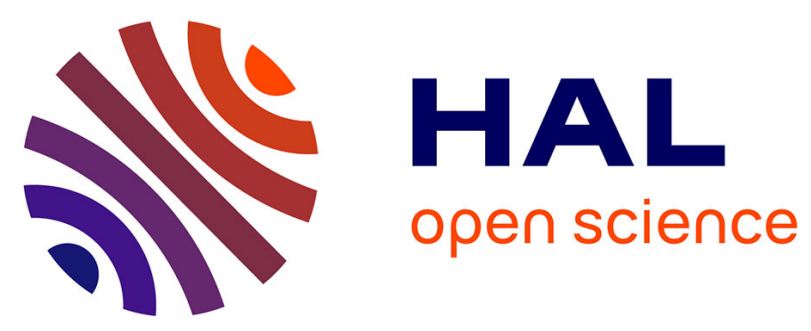

\title{
CARACTÉRISATION DE LA FRACTURATION D'UNE DEMI-SPHÈRE MÉTALLIQUE EN EXPANSION PAR ANALYSE D'IMAGE
}

Françoise Prêteux, J. Oeconomos, J. Chappelier

\section{- To cite this version:}

Françoise Prêteux, J. Oeconomos, J. Chappelier. CARACTÉRISATION DE LA FRACTURATION D'UNE DEMI-SPHÈRE MÉTALLIQUE EN EXPANSION PAR ANALYSE D'IMAGE. Journal de Physique IV Proceedings, 1991, 01 (C3), pp.C3-459-C3-464. 10.1051/jp4:1991365 . jpa-00250509

HAL Id: jpa-00250509 https://hal.science/jpa-00250509

Submitted on 1 Jan 1991

HAL is a multi-disciplinary open access archive for the deposit and dissemination of scientific research documents, whether they are published or not. The documents may come from teaching and research institutions in France or abroad, or from public or private research centers.
L'archive ouverte pluridisciplinaire HAL, est destinée au dépôt et à la diffusion de documents scientifiques de niveau recherche, publiés ou non, émanant des établissements d'enseignement et de recherche français ou étrangers, des laboratoires publics ou privés. 


\title{
CARACTERISATION DE LA FRACTURATION D'UNE DEMI-SPHÈRE MÉTALLIQUE EN EXPANSION PAR ANALYSE D'IMAGE
}

\author{
F. PRETEUX*, J.N. OECONOMOS ${ }^{*}$ et J.C. CHAPPELIER* \\ * Département IMAGE, TELECOM Paris, 46 rue Barrault, F-75013 \\ Paris, France \\ * CEA, Centre d'études de Vaujours-Moronvilliers. BP. 7. \\ F-77181 Courtry. France
}

\begin{abstract}
Résumé : A partir de clichés radiographiques de demi-coques métalliques en expansion, nous proposons une méthode de quantification de la fragmentation fondée sur les techniques de l'analyse d'image et de la reconnaissance des formes. Nous faisons coopérer ici approche texturale probabiliste, par modélisation globale du phénomène par fonction booléenne, et approche géométrique, par segmentation directe du réseau de fractures à l'aide d'une technique de lignes de partage des eaux. La méthode ainsi développée permet d'obtenir de façon fiable et reproductible la densité et la fonction de répartition surfaciques des éclats.
\end{abstract}

\begin{abstract}
From radiographic pictures of metallic half-shells in expansion, we present a fragmentation quantification method based on image analysis and pattern recognition techniques. We propose the cooperation of textural probabilistic approach (boolean function modeling of phenomena) and geometrical approach (fractures network segmentation by watersheds). So we obtain fragment mean area and distribution curve.
\end{abstract}

\section{Introduction}

La fragmentation de matériaux solides soumis à d'intenses sollicitations dynamiques a fait l'objet de nombreuses études.

Sur le plan théorique, D. GRADY, s'appuyant sur les travaux de N. MOTT $/ 1 /$,développe une théorie statistique de la fracturation dynamique et aboutit, dans le cadre monodimensionnel de l'expansion d'anneaux, à une formule analytique de la distribution en taille des fragments $/ 2 /$. Toujours sur le plan théorique, nombre de travaux étudient l'influence de la microstructure sur la naissance et le développement de la fissuration mais cela dépasse le cadre de cet article dans lequel nous nous limitons volontairement à l'aspect macroscopique de la fragmentation.

Sur le plan expérimental, les mesures concernent d'une part l'estimation de critères de rupture $/ 3 /$ et l'obtention d'une relation contrainte-déformation $/ 4 /$ et d'autre part la classification des fragments en nombre, taille, forme, surface ou masse /5/. Les diagnostics vont de I'I.D.L. (vitesses instantanées) à la récupération de fragments en passant par la cinématographie ultra-rapide intégrale ou à balayage de fente, la méthode magnétique de test de rupture, la radiographie éclair, etc.. 
$\mathrm{Si}$ les sources d'imagerie macroscopique sont nombreuses, l'analyse d'image en tant que diagnostic n'a fait son apparition que très récemment mais les résultats obtenus sont prometteurs. Nous avons en effet montré $/ 6 /$ à partir des techniques de la morphologie mathématique, que l'analyse d'une séquence de clichés optiques d'une coque métallique en expansion permet de remonter à la connaissance de certains paramètres physiques : type de déformation (purement radiale dans le cas d'une coque cylindrique), vitesse de deformation, surface moyenne d'éclat, largeur moyenne de fracture, etc..

Nous proposons ici, dans le cadre d'une approche par reconnaissance des formes, à partir de clichés radiographiques de coques en expansion, une méthode d'analyse de la fragmentation dynamique en deux étapes. La première, de nature probabiliste, se rapprochant conceptuellement de la théorie statistique de GRADY, repose sur la modélisation de texture par fonction booléenne tandis que la seconde, de nature géométrique, s'appuie sur une granulométrie après détection des lignes de partage des eaux.

\section{Modélisation texturale par fonction booléenne}

Dans le cadre de l'analyse de texture, le choix de la modélisation par fonction booléenne est justifiée par la répartition uniforme des éclats métalliques sur la surface de la calotte sphérique observée et par la vérification de l'hypothèse de stationnarité (invariance par translation) sur l'ensemble de chacun des clichés radiographiques.

\subsection{Définition d'une fonction booléenne}

Dans sa formulation la plus constructive, F. PRETEUX $/ 7 /$ définit une fonction booléenne de la façon suivante :

Soit $P$ un processus de Poisson stationnaire dans $\Pi_{0}$ d'intensite $\nu=\mu_{n} \otimes \varphi$ avec $\mu_{n}$ la mesure de Lebesgue dans $\mathbb{R}^{\mathrm{n}}$ et $\varphi$ une mesure $\sigma$-finie positive sur $\overline{\mathbb{R}}$, et soit $\left(f_{t}^{\prime}\right)_{t \in \mathbb{R}}$ une famille de fonctions aléatoires de $\Phi_{c}$ (appelées fonctions primaires). La fonction f définie par :

$f: x \in \mathbb{R} \longrightarrow \operatorname{Sup}\left(f_{t}^{\prime}(x) \oplus\{(y, t)\} /(y, t) \in P \cap \mathbb{R}^{n} \times \mathbb{R}\right) \in \overline{\mathbb{R}}$

où :

est alors une fonction booléenne stationnaire.

- $\Phi_{c}$ est l'ensemble des fonctions $f$ semi-continues supérieurement de $\mathbb{R}^{\mathrm{n}}$ dans $\overline{\mathbb{R}}$ telles que : $\exists t \in \mathbb{R} \quad S G(f) \cap \mathbb{R}^{n} \times[t,+\infty] \in K\left(\mathbb{R}^{n+1}\right)$

avec $K\left(\mathbb{R}^{n+1}\right)$ ensemble des compacts non vides de $\mathbb{R}^{n+1}$ et $S G(f)$ le sous-graphe de la fonction $\mathrm{f}$,

- П0 est l'hyperplan de cote 0 dans $\mathbb{R}^{\mathrm{n}+1}$.

- $\oplus$ désigne l'addition de Minkovski.

Ainsi, pour obtenir une réalisation de $f$, suffit-il de considérer une réalisation du processus de Poisson $P$ en chaque point $(y, t)$ duquel on fait "germer" une réalisation de la fonction primaire aléatoire $\mathrm{f}_{\mathrm{t}}^{\prime}$ dilatée par la demi-droite $[0,+\infty[$.

\subsection{Synthèse d'une texture booléenne}

Une réalisation du processus de Poisson $P$ est obtenue par un tirage aléatoire de points dans $\Pi_{0}$ selon une loi de distribution uniforme.

L'obtention d'une réalisation de la fonction primaire aléatoire est, quant à elle, beaucoup plus complexe. Nous avons choisi de déformer aléatoirement une même fonction déterministe à support compact. La plupart des auteurs limite ces déformations à des 
affinités dans des directions données de l'espace. G. BUC, lui, représente cette fonction à l'aide de fonctions B-spline cubiques dont il déplace aléatoirement les points de contrôle /8/. Les avantages de cette dernière technique sont nombreux : tout d'abord, elle assure la stabilité de l'espace de représentation dans la classe des fonctions B-spline cubiques ; ensuite, le fait que le déplacement d'un point de contrôle n'entraîne que des déformations locales de la surface correspond bien à la notion intuitive de déformation continue d'une courbe; enfin, elle bénéficie de toutes les propriétés des B-spline, en particulier leur régularité ( $C^{2}$ aux raccords).

Nous avons combiné ces deux approches afin d'obtenir le modèle de déformation le plus riche possible. Ainsi, après nous être donné le modèle de fonction B-spline primaire, contrôlons-nous ses diverses réalisations par les 5 variables aléatoires suivantes :

- les trois déplacements $(x, y, z)$ de chaque point de contrôle, représentant les variations de forme d'un éclat métallique par rapport à une forme de référence,

- un coefficient multiplicatif dans Пo, représentant une variation de surface,

- un coefficient multiplicatif suivant l'axe $\mathrm{Oz}$, représentant une variation d'épaisseur.

auxquelles nous avons imposé des lois de distribution gaussiennes de moyennes et variances données.

Afin de représenter, sans artefact d'échantillonnage, cette B-spline perturbée, nous avons choisi d'utiliser l'algorithme de CATMULL [9].

La figure 1 présente côte à côte l'image expérimentale et une texture simulée. Nous avons tenu compte de la forme des éclats (forme idéale : petit disque métallique) par l'intermédiaire d'une fonction primaire ayant la forme d'une demi-sphère aplatie. De plus, le flou radiographique est rendu par une convolution par un noyau gaussien de taille convenable.

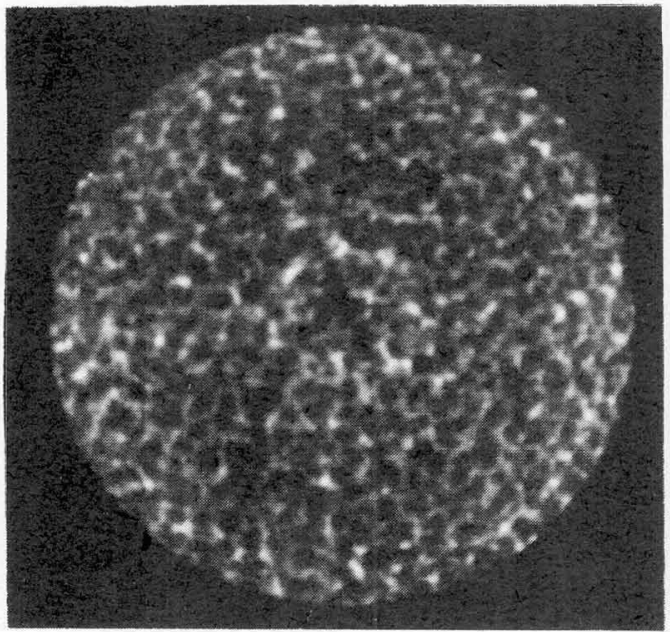

Fig. 1a

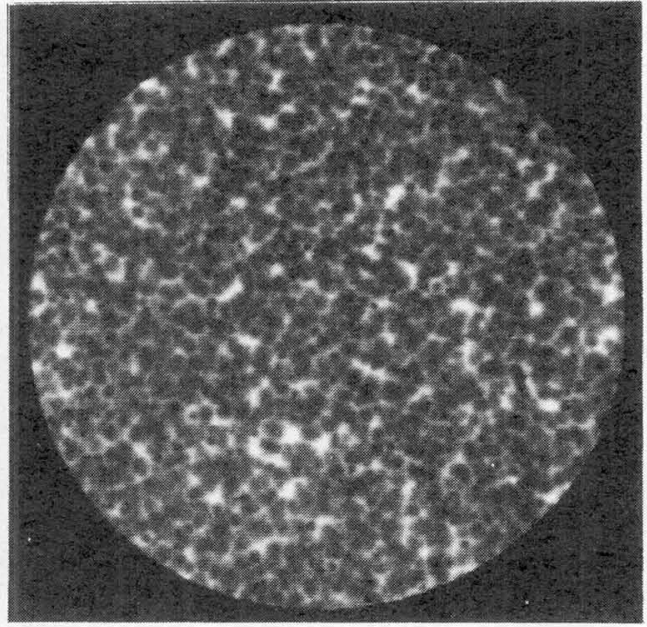

Fig. 1b

Fig. 1 - Image expérimentale (1a) et image simulée par modélisation booléenne (1b). 


\section{Analyse d'une texture booléenne}

D'une façon générale, l'analyse de texture consiste en l'extraction d'un ensemble optimal de paramètres caractéristiques. Dans le cas de textures booléennes, la thérie $\Pi /$ assure le caractère discriminant de la densité du processus ponctuel de Poisson et permet d'établir des expressions analytiques des paramètres géométriques liés à la fonction primaire.

\subsection{Aspect théorique}

De même que les fonctions aléatoires sont caractérisées par leur fonction de répartition, les fonctions booléennes stationnaires $\left(\left(\mathrm{f}_{\mathfrak{t}}^{\prime}\right)_{\mathfrak{t}}, \varphi\right)$ sont caractérisées $\Pi 7 /$ par la fonctionnelle $Q$ de $K\left(\mathbb{R}^{n+1}\right)$ dans $[0,1]$ qui à tout compact (déterministe) $K$ de $K\left(\mathbb{R}^{n+1}\right)$ associe :

$$
Q(K)=\exp \left(-\int_{\mathbb{R}} \overline{\mu_{n}}\left[\left(S G\left(f_{t}^{\prime}\right) \oplus K\right) \cap \Pi_{t}\right] d \varphi\right)
$$

Lorsque le processus ponctuel de Poisson $P$ est choisi dans $\Pi$, $Q$ s'exprime plus simplement par :

ce qui, dans le cas des images et en notant $\mathrm{K}_{\mathrm{t}}=\mathrm{K} \oplus\{\mathrm{t}\}$, mène à :

$$
\mathrm{Q}(\mathrm{K})=\exp \left\{-\mathrm{a} \overline{\mu_{\mathbf{n}}}\left[\left(\mathrm{SG}^{+}\left(\mathbf{f}^{\prime}\right) \oplus \mathrm{K}\right) \cap \Pi_{0}\right]\right\}
$$

$$
\int_{+\infty}^{-\infty} \log \left(\mathrm{Q}\left(\mathrm{K}_{\mathrm{t}}\right)\right) \mathrm{dt}=-\mathrm{a} \overline{\mu_{3}}\left(\mathrm{SG}^{+}\left(\mathrm{f}^{\prime}\right) \oplus \mathrm{K}\right)
$$

relation fondamentale pour l'analyse des textures booléennes.

En effet, le membre de gauche de cette équation peut se calculer en mesurant les différents $Q\left(K_{t}\right)$ sur l'image par :

$$
Q\left(K_{t}\right)=1-\frac{\mu_{2}\left(\left(S G^{+}\left(f^{\prime}\right) \oplus K\right) \cap(Z \odot K) \cap \Pi_{t}\right)}{\mu_{2}\left((Z \oplus K) \cap \Pi_{\imath}\right)}
$$

c'est-à-dire la surface de la troncature de cote $t$ du dilaté de l'image initiale ( $\mathrm{SG}^{+}\left(\mathrm{f}^{\prime}\right)$ ) par l'élément tridimensionnel $K$ conditionnellement à l'érodé $(Z \in K)$ du masque d'analyse Z.

Le membre de droite, quant à lui, peut être développé mathématiquement pour des compacts $\mathrm{K}$ convenablement choisis. En particulier, si $\mathrm{K}$ est un segment de longueur $\lambda$, on a :

$\overline{\mu_{3}}\left(\mathrm{SG}^{+}\left(\mathrm{f}^{\prime}\right) \oplus \mathrm{K}\right)=\lambda \mathrm{S}\left(\mathrm{SG}^{+}\left(\mathrm{f}^{\prime}\right)\right)+\overline{\mu_{3}}\left(\mathrm{SG}^{+}\left(\mathrm{f}^{\prime}\right)\right)=\lambda \mathrm{a} 1+\mathrm{a} 0$,

et si $\mathrm{K}$ est une sphère de rayon $\rho$, on obtient :

$\overline{\mu_{3}}\left(\mathrm{SG}^{+}\left(\mathrm{f}^{\prime}\right) \oplus \mathrm{K}\right)=(4 \pi / 3) \rho^{3}+\rho^{2} \mathrm{M}\left(\mathrm{SG}^{+}\left(\mathrm{f}^{\prime}\right)\right)+\rho \mathrm{S}\left(\mathrm{SG}^{+}\left(\mathrm{f}^{\prime}\right)\right)+\overline{\mu_{3}}\left(\mathrm{SG}^{+}\left(\mathrm{f}^{\prime}\right)\right)$,

$$
=a 3 \rho^{3}+a_{2} \rho^{2}+a 1 \rho+a o
$$

où $S(X)$ et $M(X)$ désignent la surface de $X$ et l'intégrale de la courbure moyenne de $X$.

\subsection{Aspect pratique}

Pratiquement, il en découle que "le volume des dilatés linéaires (resp. sphériques) successifs d'une texture booléenne a un comportement linéaire (resp. cubique)" $7 /$. Ainsi, en considérant comme compact $\mathrm{K}$ des sphères (resp. segments) de taille croissante et en mesurant à chaque fois le volume du dilaté $\int_{+\infty}^{-\infty} \log \left(\mathrm{Q}\left(\mathrm{K}_{\mathrm{t}}\right)\right) \mathrm{dt}$, obtient-on un nuage de points définissant une cubique (resp. droite) dont les coefficients a0, a1, a2 et a3 (resp. ao et al) sont directement reliés aux paramètres caractéristiques de la fragmentation : densité d'éclats, surface moyenne et volume moyen de la fonction primaire.

Les résultats obtenus par cette méthode d'analyse sur les clichés de demi-sphères en 
expansion sont discutés au paragraphe 5 .

\section{Ligne de partage des eaux et granulométrie}

Une approche complémentaire de la précédente, non plus probabiliste mais géométrique, consiste à s'intéresser directement au réseau des lignes de fracture.

Les fractures, correspondant à un manque de matière au niveau de l'objet, sont des lieux d'absorption minimum sur le plan radiographique et se traduisent donc au niveau de l'image par des lignes de crête du signal. C'est pourquoi la technique de ligne de partage des eaux (L.P.E.) /10/ nous paraît la mieux adaptée à leur détection.

Considérons, en effet, l'image comme un pseudo-relief. Une goutte d'eau placée en un point quelconque de ce relief descendra suivant la ligne de plus grande pente jusqu'à ce qu'elle ait atteint un exutoire (minimum régional). L'ensemble des points du relief menant au même exutoire est appelé bassin versant et la L.P.E. est la ligne séparant au moins deux bassins versants différents /11/. Notons qu'en raison de la sensibilité au bruit de l'algorithme de détection des L.P.E. d'une image, nous avons effectué un filtrage morphologique préalable afin de garantir la robustesse de l'extraction du réseau de fractures (figure $2 \mathrm{a}$ ).

L'image des éclats correspondant aux bassins versants est alors définie comme le complémentaire, au sens ensembliste, de la L.P.E.. Une granulométrie $/ 12 /$, ici une famille croissante d'ouvertures morphologiques, effectuée sur l'image des bassins versants détermine ainsi une répartition en forme et taille des éclats (figure $2 b$ ).

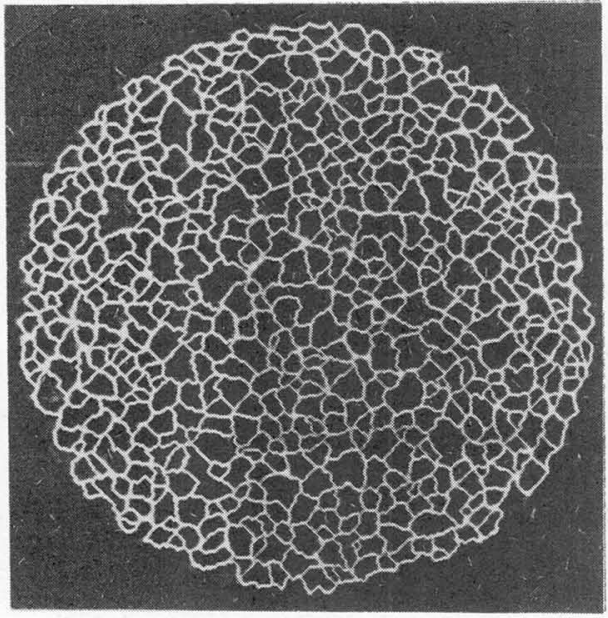

$2 a$ : L.P.E. de 1 a filtrée.

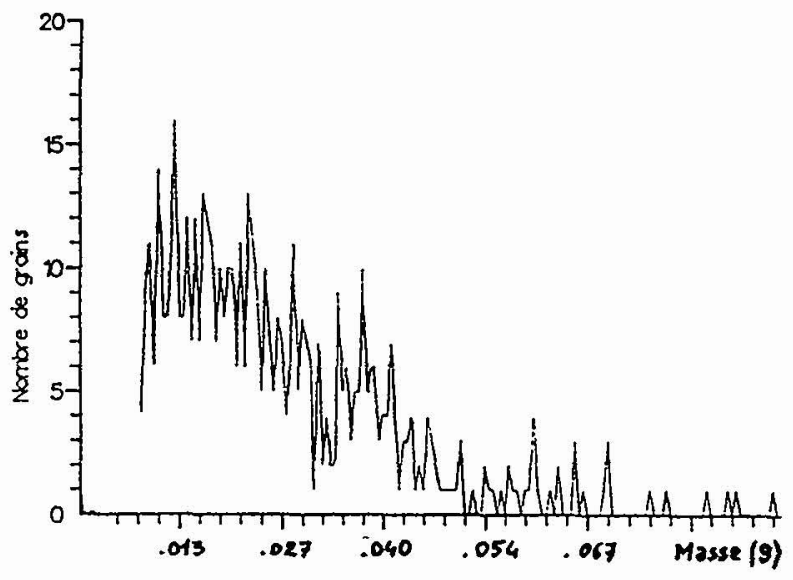

2b : Granulométrie de 2a complémentée.

Fig. 2 - Analyse de la fragmentation par extraction de L.P.E.

\section{Résultats - Discussion}

Les deux méthodes précédemment décrites permettent de quantifier :

- la densité d'éclats (nombre d'éclats par centimètre carré),

- la fonction de répartition des éclats.

L'analyse par modélisation texturale booléenne nous donne une densité du processus ponctuel de Poisson sous-jacent de 36, donc une surface moyenne d'éclat de $0,027 \mathrm{~cm} 2$ 
(inverse de la densité) et une masse moyenne d'éclat de l'ordre de $0,02 \mathrm{~g}$ (tous les résultats sont à $5 \%$ près).

L'analyse granulométrique du réseau de L.P.E. fournit une fonction de répartition semblable à celle présentée en $15 /$ pour de l'uranium. Son aspect bruité est dû au fait que nous n'observons qu'une petite partie de la surface de la demi-sphère (calotte sphérique de demi-angle $\pi / 4$ ) ce qui dégrade la statistique.

Remarquons qu'en l'état actuel de nos recherches, nous n'avons pas encore exploité l'ensemble des mesures fournies par la méthode développée, nous étant limités aus grandeurs physiques les plus simples.

\section{Conclusion}

La coopération de deux approches d'analyse d'image, l'une probabiliste et l'autre déterministe, se révèle particulièrement constructive pour fournir un diagnostic quantitatif pour caractériser la fragmentation de demi-coques métalliques en expansion de façon simple et reproductible.

\section{Remerciements}

Que R. DORMEVAL trouve ici le témoignage de notre reconnaissance pour l'intérêt qu'il a bien voulu porter à nos recherches.

\section{Références}

/1/ MOTT N. F., Proc. Royal Soc. London, A189, 300 (1947).

12/ GRADY D. E., Shock waves and high-strain rate phenomena in metals, pp. 181-192, Plenum Press, New-York, 1981.

13/ OLIVE F., NICAUD A., MARILLEAU J., LOICHOT R., Mechanical properties at high-strain rate 1979, pp. 242-251, Inst. Phys. Conf. Ser. N 47, Chap. 2.

14/ WARNES R. H., DUFFEY T. A., KARPP R. R., CARDEN A. E., Shock waves and high-strain rate phenomena in metals, pp. 23-36, Plenum Press, New-York, 1981.

15/ STELLY M., LEGRAND J., DORMEVAL R., Shock waves and high-strain rate phenomena in metals, pp. 113-125, Plenum Press, New-York, 1981.

16/ OECONOMOS J.-N., CARNOT M., SCHMITT M., "Analyse de la fracturation d' un cylindre métallique en expansion rapide par la morphologie mathématique", In Proc. Symposium on High Dynamic Pressure, La Grande Motte, France, 1989.

7) PRETEUX F., "Description et interprétation des images par la morphologie mathématique. Application a l'imagerie médicale", Thèse de doctorat ès sciences, Universite Paris VI, octobre 1987.

18/ BUC G., "Analyse et modeles de textures : état de l'art", Mémoire de fin d'études, Département IMA, Télécom Paris, 1990.

19/ CATMULL E., "A subdivision algorithm for computer display of curved surfaces", $\mathrm{Ph} . \mathrm{D}$. Thesis, University of Utah, December 1974.

/10/ DIGABEL H., LANTUEJOUL Ch., "Iterative algorithms", In Proc. Second European Symposium on Quantitative Analysis of Microstructures. J.L. Chermant Ed., Riederer Verlag, Stuttgart, pp. 85-99, 1978.

/11/ PRETEUX F., MERLET N., "New concepts in mathematical morphology : the topographical and differential distance functions", In Proc. SPIE, July 21-26 1991, San-Diego, U.S.A..

/12/ MATHERON G., "Random Sets and Integral Geometry", Wiley\&Sons, New York, 1975. 